\title{
CULTURAL HERITAGE AND DIGITAL TOOLS: THE ROCK INTEROPERABLE PLATFORM
}

\author{
B. TURILLAZZI, G. LEONI, J. GASPARI, M. MASSARI \& S. O. M. BOULANGER \\ Department of Architecture, University of Bologna, Italy
}

\begin{abstract}
The digitisation of urban cultural heritage $(\mathrm{CH})$ is recognised within EU (European Union) policies as an opportunity to make $\mathrm{CH}$ a driver for urban transformation towards a sustainable and inclusive future. Various digital platforms are emerging as tools not only to store, retrieve, compare and process different kind of data related to $\mathrm{CH}$ for the use of urban planners and administrators, but also as participative tools for distributed decision-making.

The increasing integration between the physical and digital realm through various digital instruments such as the Internet of things, virtual and augmented reality, machine learning and natural language processing, has led designers to conceptualise the necessity of merging different smart city dashboards and platforms into an integrated system known as the urban digital twin (DW). This task can be made possible only through the construction of a shared ontology of the city, which allows the interoperability of different data systems.

The DWs, originally developed in mechanical and process engineering, allow to construct a digital model of a physical object or process, to monitor its real-time performance, to perform maintenance tasks and to test the effects of planned changes. However, when extending the notion of the DW to complex cultural and social entities such as cities, it is important to consider also issues of inclusivity and citizen participation. How can the DW be conceptualised not only as a tool of technological control or narrative, but as an instrument to empower not only institutions but also citizens? The experience gathered in the construction of the ROCK platform and its participatory ontology, developed within the Horizon 2020 (H2020) funded project ROCK (GA 730280), becomes an important precedent in this task. Keywords: collaborative platforms, cultural heritage digitalisation, cultural heritage-led regeneration, digital twin, living lab
\end{abstract}

\section{INTRODUCTION: THE TRENDS OF CULTURAL HERITAGE DIGITISATION}

European cities are undergoing major changes, including increasing urbanization and technological advances further urged by the contingency caused by the Covid-19 pandemic. The recovery from the pandemic spurs EU countries to develop measures that make societies inclusive and communities more resilient. Incorporating resilience, adaptation in urban development is key: it is from cities' stratifications and legacy that a new model of development and resilience can start. In this scenario, historic cities and cultural heritage $(\mathrm{CH})$ hold both the legacy and the stratified information: keys to sustainable and inclusive growth through urban regeneration processes [1-3]. Meanwhile, the pandemic accelerated the momentum of $\mathrm{CH}$ digitization, which has long been encouraged by EU policies and supported by local political engagement. Policy measures have been oriented to urge the commitment by Member States [4], also envisaging a reflection with respect to the bureaucratic and regulatory aspects of the digitization.

A more informed $\mathrm{CH}$-led access could represent an asset to start building a different vision and narration for cities [5]. In addition to preservation of physical $\mathrm{CH}$, timely steps need to be taken for gathering, managing and easily distributing information and data pertaining tangible and intangible assets of cultural value. This is even more true in the aftermath of the Covid-19 outbreak, which saw on the one hand a drastic reduction of direct experience of culture and on the other a heavy transition towards digital replicas of $\mathrm{CH}$ fruition. 
In the constellation of tools and technologies aimed at enabling the access to $\mathrm{CH}$ [6], what is still to be investigated is the possibility to overcome critical issues such as broader access to citizens and interaction with institutions in $\mathrm{CH}$ sector, as requested by the Digital Cultural heritage NETwork [3]. This would be eased by defining an interoperable system, addressed to technicians, professionals, policy makers, scholars but also end-users, investors and citymakers. The hypothesis of the paper is that such technological tools must be equipped with an interactive interface that dialogues both with designers and less-expert users, in a way that it is not just technocratic and narrative, but inclusive and open to divergencies. The idea is that such an approach would prevent the risk to miss the critical interpretation of the data belonging to the physical dimension of the city and its $\mathrm{CH}$ which, with their unpredictability, constant and dynamic changes, appears difficult to be perceived by a virtual simulated environment.

These considerations constitute the grounds for the reflections and experiments of the ROCK project - funded under the Horizon 2020 program (GA 730280) - a peculiar example of an integrated approach aimed at addressing the need to make real-time data available for broader uses, providing new applications to facilitate access to $\mathrm{CH}$. The platform developed by ROCK brought together new civic knowledge enhancing the collaboration across disciplines, $\mathrm{CH}$ experts and users, social and technical actors.

\section{OBJECTIVES AND METHODOLOGY}

The aim of the paper is to critically investigate the possibilities offered by heritage digitization, its data accessibility and the usability of its technical and narrative elements. The starting assumption is that accessibility to $\mathrm{CH}$ is guaranteed if it includes citizenship among its users, not only as a mere recipient, but also as a co-producer of useful data, as holder of usable knowledge. The digital twin (DW) dimension appears useful to verify against the case study of the ROCK interoperable platform, set up as an environment of collaboration between urban actors, but also as a repository of co-created data on $\mathrm{CH}$.

After an introduction on the current trend in $\mathrm{CH}$ digitization against European policy orientations, and a methodological overview, the paper explores some cases of $\mathrm{CH}$ digitization though collaborative platforms and DWs in EU projects. This framework allows to highlight the necessity to go beyond the DW as a tool of technological control or narrative, but as an instrument to empower both institutions and citizens. The ROCK interoperable platform is presented as a useful case study that operated this integration by making use of enlarged ontologies of data processing to extend the access to $\mathrm{CH}$ usable knowledge. The final paragraph concludes highlighting some lessons learned from ROCK and putting forward the presented case study as a valuable testbed for future $\mathrm{CH}$ policy concretization, in the framework of the Green Deal and the NEB implementation.

The first part of the paper provides a qualitative review of the main EU policy orientations towards $\mathrm{CH}$ digitization through a desk research in the main EU repositories (Cordis, OpenAire, EU innovation packs). The same desk and literature review was performed for the following part of the paper, concerning the role of the DWs. Thanks to a direct involvement of the researchers in the project, the ROCK case study was investigated with personal notes, and direct observation.

3 STATE OF THE ART OVERVIEW: CH DIGITIZATION AND DIGITAL TWINS "[...] there are times when science can seem to lose its connection to society and its needs, and sometimes its objectives are not fully understood, even if they are well intended. The lack of a common language and rapid progress in many areas of research has increased the 
public's concern or contributed to ambivalence about the role that science and technology play in everyday life. But science cannot work in isolation, and advances in science and technology are not an objective in their own right" [7].

This approach, also known as "Science with and for Society" stresses the importance of reducing the distance between technical-scientific knowledge and the wide public. In the field of $\mathrm{CH}$ studies, this approach seems to be more understandable as the public is active part of the common stratification and sense of belonging of a place. However, digital instruments can overcome the passive interaction towards $\mathrm{CH}$ by turning users into prosumers, creating new knowledge, fostering innovation [8], being levers of socio-economic development $[9,10]$.

$\mathrm{CH}$ is understood as an unlimited source of stratified information, which need to be released to build conscious design and planning for the cities of the present and future [11]. This information needs to be returned and made clearly available to all urban agents who can implement skills to enhance its value, and to those who can benefit from it.

The chance offered by $\mathrm{CH}$ digitization, is also to provide cultural private and public institutions, with opportunities to innovate the preservation and management of cultural resource, and to grant broader access to knowledge and data on $\mathrm{CH}$. According to Owens [12] in fact, the most important reason to digitize $\mathrm{CH}$ data is to grant a multitude of users with the ability to explore and connect with the legacy of the past, while at the same time measuring how people use, reuse, explore and understand $\mathrm{CH}$.

The signature of the "Declaration of Cooperation on Advancing Digitization of Cultural Heritage" during the Digital Day in 2019 and the work started since the European Year of Cultural Heritage in 2018, were first collective steps undertaken by the Member States to commit to the digitization of $\mathrm{CH}$ and to strengthen its widespread accessibility. Similarly, the recent "Declaration on joining forces to boost sustainable digital transformation in cities and communities in the EU" [13] signed by 86 representatives of multi-level public administrations, set the role of the digital transition as a way to "help to create places where people enjoy living and working" especially in times of crisis.

This tendency was recently confirmed as a priority in the New Green Deal, where EU recalled the importance of harnessing the "power of data" [14] offering immense potential to drive climate action and support a just transition to a resilient future. There are significant cultural dimensions to every aspect of the New Green Deal, from circular economy to building renovation, to boosting communities' resilience via legacy and situated knowledge. The intersection between $\mathrm{CH}$ and climate transition is reinforced [15] overcoming the tendency to keep the two issues separated in research and policymaking. The study and valorization approach to $\mathrm{CH}$ - which is mostly narrative and qualitative [16] - can now expand with quantitative data collection, necessary in acting against climate change. $\mathrm{CH}$ becomes a source of true resilience as claimed in the New European Bauhaus (NEB) EU initiative, merging art and science in a "collaborative creation space between various disciplines and stakeholders" [17]. NEB aims to take advantage of the digitization revolution that has long marked European $\mathrm{CH}$ research and will be reinforced in the future, as emerging in the Work Plan for Culture 2019-2022 and in the New European Agenda for Culture.

\subsection{The objectives of $\mathrm{CH}$ digitization: the perspective of the Science with and for Society}

The growth of innovative technologies to store historicized data, but also the monitoring of processes affecting $\mathrm{CH}$, do not only collect but also generate new data from diversified sources. DWs, IoT data, augmented reality, AI (Artificial Intelligence), on-line archives [18], and digital collaborative platforms, management dashboards and monitoring sensors [19-21], 
can provide new opportunities for the "creation of new spaces, not only for consumption of heritage but also for its critical reflection" [22].

These directions of research put great attention to opening data and information on the management of various $\mathrm{CH}$ assets to scholars, researchers, professionals, curators as well as decision and policymakers. Several digital platforms to manage and interact with $\mathrm{CH}$ have been working in this direction in the last decades. Among them the text presents three examples, for their soundness and their services aiming to foster interactions with a broader non-expert audience.

This is the case of Open-Heritage.eu, a platform created by the REACH project ${ }^{1}$ (RE-designing Access to Cultural Heritage for a wider participation in preservation, (re-)use and management of European culture) and including the work of other projects such as RICHES and MEMOLA. ${ }^{2}$ Here the heritage community (being it researchers, practitioners, professional or just citizens) can interact in the digital environment to build contribute to its enhancement, by participating to the scientific debate, or suggesting case studies or even co-working on a common taxonomy on $\mathrm{CH}^{3}$

Another example is the Innovators in Cultural Heritage Platform ${ }^{4}$ created by the ROCK project together with the MARINA ${ }^{5}$ one. This digital space targets innovators in $\mathrm{CH}$ and it offers them the possibility to build a community, by sharing workspaces, materials, news and events.

A third example is provided by the UNESCO "Dive into Intangible Cultural Heritage" interactive navigation. Here people can explore the different domains of intangible cultural heritage discovering it through their categorisation. The platform builds its content on a web-semantic base, grouping elements in unconventional ways. This approach is currently under enhancement by the iHERITAGE project, ${ }^{6}$ that aims at incorporating innovative technologies to understand how to enhance participation in the UNESCO $\mathrm{CH}$ domains.

These few examples of the growing tendency of building platforms to interact with $\mathrm{CH}$ various aspects. However, what seems to be lacking are systems able to further enhance citizens participation. This is the road paved by DWs, that if applied to $\mathrm{CH}$ can provide an enhanced way to turn people into active co-creators of contents, projects, data concerning the renovation, valorization and cultural processes.

\subsection{Digital twins as an enabling community tool}

Among this escalation of tools and devices [23], the DWs [24] (DTs) are growing in interest, for their capacity to build engagement among citizens in an easy way and to provide an effective instrument for gathering data to inform new planning paths. As stated in the literature, DWs can be intended as a representation of the reality in a virtual environment [25] where different targets can perform actions based on the digitalisation of the reality. This can be done in the form of spatial redesign of the real environment but also through a data representation. Although first imagined by a computer scientist, it is in the context of the

\footnotetext{
$\overline{{ }^{1} h t t p s: / / w w w . r e a c h-c u l t u r e . e u / ~}$

${ }^{2}$ https://www.riches-project.eu/; https://memolaproject.eu/;

${ }^{3}$ https://www.open-heritage.eu/riches-taxonomy/

${ }^{4} \mathrm{https}: / / \mathrm{www}$. innovatorsinculturalheritage.eu/

${ }^{5} \mathrm{https}: / /$ www.marinaproject.eu

${ }^{6} \mathrm{http} / / /$ www.enicbcmed.eu/projects/iheritage
} 
manufacturing sector that such concept most rapidly develops: "The Digital Twin is a set of virtual information constructs that fully describes a potential or actual physical manufactured product from the micro atomic level to the macro geometrical level. At its optimum, any information that could be obtained from inspecting a physical manufactured product can be obtained from its Digital Twin" [26]. DTs are envisioned as tools enabling the implementation of complex prognostics and intelligent maintenance, to benefit the productivity of an industrial plant. In this definition, and in such scope, we can also identify the initial constituents of DTs, i.e., computational structures such as computer models, simulation paradigms and any data describing their real instances and interfaces with the physical world.

The concept of digital twin has been transferred to urban studies for its potential in creating collaborative planning processes, and in enabling participants with diverse backgrounds to achieve an agreement [27,25]. As set by Dembski [28], DTs can be used for analysing potential impacts of urban changes, giving the possibility to citizens to learn and contribute both to the transformation of spaces and to the understanding of impacts, since the earliest stages of the process. Although the state-of-the-art research in the domain of DTs is progressively integrating technologies that are related to the production, management and processing of data (i.e., Internet of Things, Cloud Computing and Artificial Intelligence), other directions are also being sought. In addition to the need for automation, also a need for human collaborations and direct interventions is emerging. This may be noted with the integration of immersive solutions, on one side, and collaborative ones, on the other [29].

To this extents, DW platforms have been used to exchange experiences and collaboratively seek for solutions [30]. These platforms have placed high emphasis in defining an interoperable system, based on a single platform, able to integrate, address and exchange [31] information among technicians, professionals, policy makers, scholars, but also end users, investors and city-makers. This is, for example, the case of the Florence Smart City Control Room, developed by the European project Snap4City ${ }^{7}$ where a large amount of big data is collected and distributed in open source to everyone interested, citizens or technician. In this example, the platform allows people to see data, also on a georeferenced way, and to create personalized visualizations and graphs.

According with the recent "Declaration on joining forces to boost sustainable digital transformation in cities and communities in the EU" [13] and the launch of the living-in.eu platform, some specific features are identified for effective DTs. In particular, five features that the DW should have: being connected, being integrated, giving the possibility to visualize elements and to perform analysis and, finally, being secure. However, these platforms still encounter challenges to be solved, such as data quality and availability, semantics and shared vocabularies, data governance, ethics and privacy.

Adopting a design-for-all approach in the deployment of collaborative platforms, might represent the innovation potential for $\mathrm{CH}$-led regeneration, increasing the accessibility of contents, the openness, the user-friendliness of the interface, the enabling factors deriving from the adoption of immersive technologies at urban scale. In particular, the ROCK (Regeneration and Optimisation of Cultural heritage in creative and Knowledge cities) H2020 project proposes an "enabling platform" [30,31] for the knowledge, management, monitoring, redevelopment and enhancement of $\mathrm{CH}$. It addressed some of the current issues and ambiguities related to $\mathrm{CH}$ digitisation elements proposing a shared vocabulary for digital heritage through its interoperable platform ecosystem.

${ }^{7}$ https://www.snap4city.org/ 


\section{THE CASE OF THE ROCK INTEROPERABILITY SYSTEM}

ROCK - Regeneration and Optimisation of Cultural heritage in creative and Knowledge cities $^{8}$ is a project funded by the European Union's Horizon 2020 research and innovation programme under Grant Agreement No. 730280, and the call "Greening the Economy". The consortium was coordinated by the Municipality of Bologna and included 32 partners from 13 countries. Its activities were deployed from 2017 to 2020. The project focused on historic city centres as laboratories to demonstrate how $\mathrm{CH}$ can be an engine of regeneration, sustainable development, and economic growth [32]. ROCK approach foresaw the systemic and integrated application of a series of role-model initiatives in three testing sites in the cities of Bologna, Lisbon and Skopje, supporting the transformation of historic city centres afflicted by physical decay, social conflicts and poor life quality into Creative and Sustainable Districts. The project goal was to provide a collaborative approach $[33,34]$ to promote the effective regeneration and adaptive reuse in historic city centres by implementing a repertoire of successful CH-led regeneration initiatives [35]. ROCK moved beyond a specialized idea of $\mathrm{CH}$ understood exclusively in terms of preservation. Instead, $\mathrm{CH}-$ especially in its public and unused spaces - is seen as an incremental and changing store of value that can drive both spatial and behavioural change. This change occurs in ROCK through the implementation of a Living Lab methodology [36] in the three demonstration areas: pop-up architectural interventions, small installations, ethnographic and cartographic observations, were performed in collaboration with a large plurality of local actors among which are cultural operators, local resident, city users, students and institutions. The Living Lab aimed at experiment, observe and verify a palimpsest of actions for the enhancement of accessibility to services and $\mathrm{CH}$ goods and for strengthening collaborations among a plurality of actors. The demonstration area gathered a set of structural data on $\mathrm{CH}$ contextual conditions (e.g. number of heritage assets, museums, libraries), enriched with dynamic ones (e.g. flow of users visiting an artistic installation, permanence in a regenerated public space) collected from sensors and from qualitative information emerged from questionnaires, surveys and ethnographic observations. Local datasets were collected with the goal of re-defining and improving the Living Lab interventions, to eventually inform policy and decision-makers with the knowledge acquired, as evidence both of the effectiveness and of the failures associated with each initiative. The replicability of knowledge and lessons learned from data mining and analysis, aimed at developing informed integrated management plans for the three historic areas, with associated funding mechanisms.

The set of various sources, dynamic qualitative and quantitative data, ethnographic and social observations, were held together by a digital infrastructure, an interoperable platform that brought together both structural data and dynamic ones, as well as information that emerged from participatory activities and explorations. The ROCK platform worked on the relational aspects of $\mathrm{CH}$ recognition, production and management, providing procedures to design, monitor and evaluate $\mathrm{CH}$-driven urban regeneration processes that include and intertwine not only measurable data, but also information emerging from field observations, interviews, ethnographic research and participatory activities.

The ROCK platform ${ }^{9}$ is a decision-support tool not only meant to be informational or managerial but aimed at enabling the observation and evaluation of a novel approach to $\mathrm{CH}$ regeneration in Europe's historical centres. The following section clarifies the methodological

\footnotetext{
${ }^{8}$ https://rockproject.eu/

${ }^{9}$ https://opendata.rockproject.eu/rock/
} 
approach used by ROCK in Bologna to identify datasets, collect them, and build the most useful interrelationships to generate a shared language for $\mathrm{CH}$-driven regeneration.

\subsection{Methodology of data collection and processing in ROCK Bologna}

ROCK's approach aimed to open up the possibility of thematic and systematic access to CHrelated data, overcoming its constraints and limitations and providing possibilities for new uses.

The peculiarity of ROCK approach lies in the definition of the data collection and monitoring strategy: the identification of the relevant datasets to be collected and of the key performance indicators were in fact co-designed locally.

Each pilot site first identified an ecosystem of local stakeholders, representatives of different institutions, cultural producers, SMEs and citizens' associations involved in the cultural life of the demonstration area. In Bologna, the ROCK experimentation area was located in the historical centre of the city, in the university area. In Bologna, the Living Lab model was applied via the Living Lab, an iterative research-action initiative, working on place-based accessible solutions, proposing tactic interventions, both oriented towards behaviour change and space modification.

The local ecosystem of stakeholder of the Living Lab was involved in verifying the situated needs of the community, but also in defining the requirements to be met for the evaluation of actions related to the regeneration process. The ecosystem, the researchers, and the public administration staff collaborated to define three scenarios for transforming the pilot area: "sustainability," "accessibility," and "collaboration for new cultural productions" as general thematic axis, eventually contextualized in selected locations in the university area. The three contextual definitions of the scenarios and a set of requirements to be met, were linked to the relevant key performance indicators.

These inputs were incorporated as datasets and added up to the dynamic and structural datasets collected in the interoperable platform. Examples of datasets included in an aggregated form in the platform (accessible in open source in the opendata.rockproject.eu) are data related to ROCK sensors and tools (i.e. people flow analytics, microclimate related data, etc.) but also results of the Living Labs in form of pdf reports.

The activities of the Living Lab kept together the construction of a shared, situated and local understanding of $\mathrm{CH}$, produced through bottom-up and shared processes, with the imperative of the construction of an interoperable system based on universal ontologies. This way, ROCK produced a first shared and local ontology of CH-led urban regeneration in the university district of Bologna. In order to ensure interoperability, the ROCK ontology produced, was translated using existing standard ontologies for $\mathrm{CH}$ (e.g. the Getty Institute Art and Architecture Thesaurus, Link Geo Data Ontology, Dbpedia Ontology), tourism and points of interest, smart city sensors (e.g. SSN, Semantic Sensors Network Ontology). This has transformed the three principles of the ROCK project into inspiring design lines and interpretative matrices for the monitoring of public affluence in the area, its appreciation and access to previously neglected $\mathrm{CH}$. The Bologna datasets, the georeferenced participatory mapping, the requirements of the area provided by the participatory activities, were eventually used as resources for the development of an App-BOforAll ${ }^{10}$ - for the exploration of accessible itineraries in the university area of the city. The interoperable platform has given substance to this process.

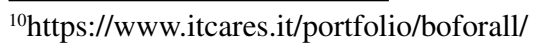




\subsection{The ROCK interoperable platform as a digital twin}

The ROCK interoperable platform allowed the collection, processing and comparison of data by facilitating communication and relationships between various sources of information. The activities of the Living Lab provided the general horizon through which a variety of data could be interpreted. The ROCK platform, became the tool through which a heterogenous variety of datasets could be stored, retrieved, visualised, analysed and elaborated according to the conceptual categories individuated in the participatory and consultation phase with stakeholders.

The selection of datasets was made according with the specificities of $\mathrm{CH}$ but also considering some of the most pressing issues that cities need to face, such as climate change, resilience, pollution. In particular, the identification of the specific data, with unit of measurement and standards has been performed during the first year of the ROCK project, through the creation of a task force of researcher, stakeholders, public administrations staff, technological partners. The task force identified some major key aspects, or thematic layers, to be investigated through the project, via different datasets (e.g. microclimate related dataset through the detection with local sensors of air temperature, air humidity and wind speed; air pollution dataset based on local sensors.

The datasets included in the ROCK platform were then grouped in the following main typologies:

- historicized datasets, already available from public institutions or private companies, such as points of interest, museum and theatre attendance, free-floating bike sharing services data;

- datasets related to ROCK activities, collected through the monitoring of activities directly on site, and communicated through connected ROCK platforms, such as the ROCK Atlas ${ }^{11}$ and ROCKME ${ }^{12}$;

- datasets from real-time sensors tested as part of the ROCK project (temperature, humidity, noise, pollution, wind, flow and video neuroanalysis);

- qualitative datasets from specific analyses and activities [41], including event mapping, sentiment analysis related to rock actions, surveys, ethnographic research, etc.

Nevertheless, the ROCK platform system is extensible and scalable to store and elaborate different types of data.

Its structure is composed by a variety of interfaces, which allow various kinds of users and interests' groups to interact with it, according to their IT proficiency and interest. Firstly, a data storage interface, based on CKAN and linked to the open data platform of the municipality of Bologna, allows users to upload and retrieve the datasets listed above. Then, a business intelligence platform provides dashboards which could by customised by the end user to visualise and compare the various datasets on temporal, relational or spatial (map) interfaces. A linked open data interface allows to navigate through the data stored in the platform, whereas a SPARQL endpoint, meant for experienced users, data scientists and researchers, allows to extract data according to user-defined queries.

\footnotetext{
${ }^{11} \mathrm{https}$ ///atlas.rockproject.eu

${ }^{12} \mathrm{https}: / /$ opendata.rockproject.eu
} 


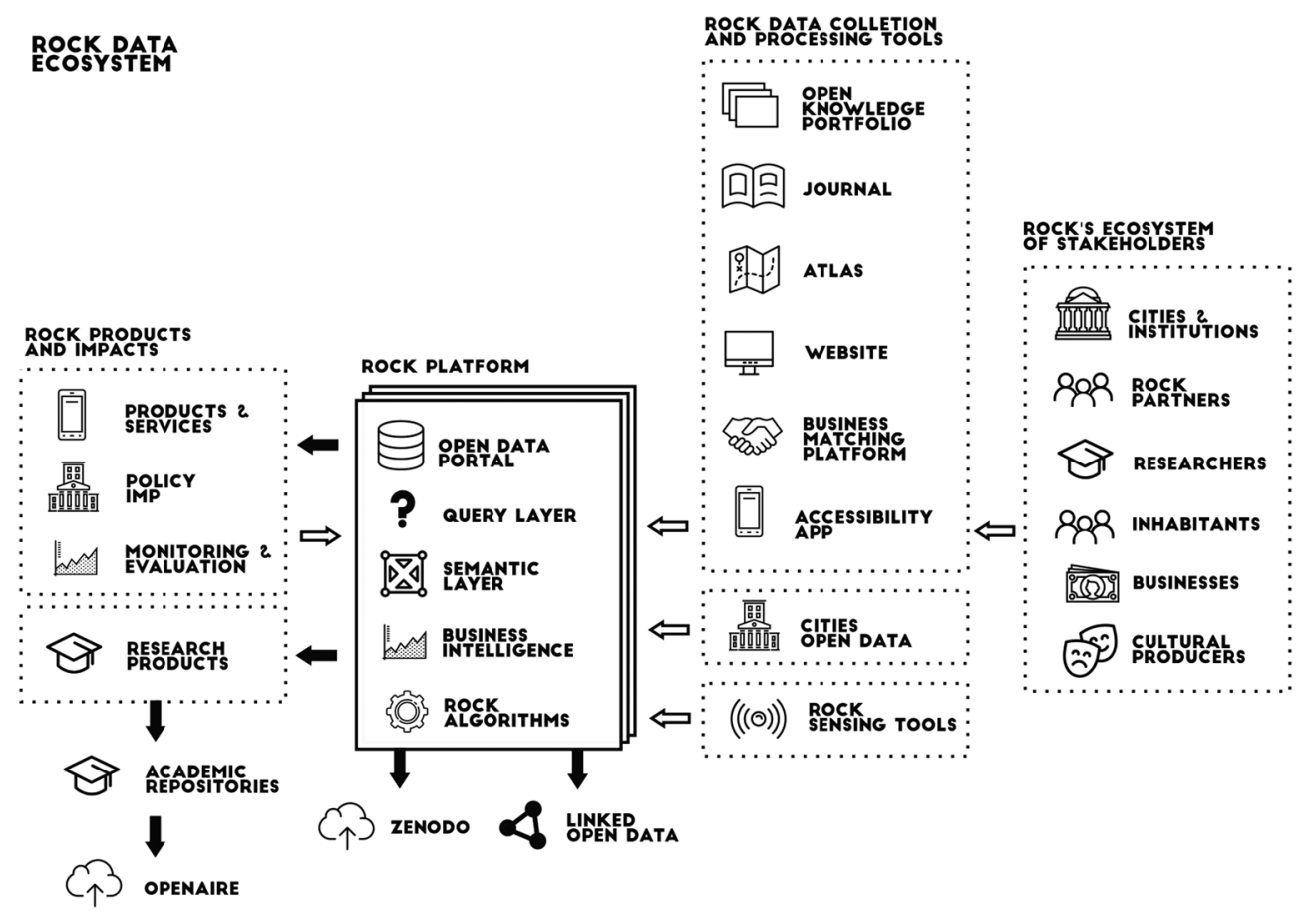

Figure 1: ROCK interoperable platform and data ecosystem. Artwork by Amir Djalali, 2019.

Considering the current debate on DWs in the scientific domain, the ROCK interoperable platform acquires a particular importance. It shows promising results in almost all five features foreseen for the DW and recalled in the previous paragraphs. It is connected and integrated, thanks to the complex interoperable ecosystem put in place; it gives the possibility to show results by visualising the elements, with infographics directly in the platform and as geo-localised information, thanks to its direct link to an online Atlas; it gives the possibility to perform analysis although this aspect is still to be refined and automated; it is secure, since it follows data protection management and ethics protocols for data and information protection.

The tools developed and tested within ROCK to verify and measure the actions impact, dealt with different kind of sources and were aimed at different audiences. ROCK interoperable platform, allowed these data to be gathered, accessed, visualised and compared, facilitating communication and relationships between different information sources.

The platform defined a common ontology on $\mathrm{CH}$, enabling a transition from a segmented and technocratic approach to management, to a circular and inclusive one of collaboration. ROCK's digital ecosystem reconnected all the actors involved in the process of $\mathrm{CH}$-driven regeneration, allowing the exchange of information through the development of a conceptual grid of historical data, empirical evidence and dynamic monitoring results, to observe and validate the process.

\section{CONCLUSIONS}

The ROCK project proposed a repository and an operative framework for processing a range of data and information both historically collected, dynamically gathered and derived from 
the consultation with actors involved during the research activities, through an interoperable database. Its outcomes in terms of data produced and results achieved can be read in different interpretative domains.

The way in which the ROCK platform approaches the issue of digitization of $\mathrm{CH}$ goes beyond the idea of merely preserving physical cultural assets and making them accessible through digital platforms. On the contrary, $\mathrm{CH}$ is looked after as a living and transforming set of practices, of dynamic contingencies and uses, which allows to turn it into an operative tool, as a driver for urban transformation. Hence, ROCK foresees a new role for digitized $\mathrm{CH}$. On the one hand, data collected and visualised show evidence of the ability of $\mathrm{CH}$ to transform and adapt to contingent dynamics, on the other, the digitization of $\mathrm{CH}$ allows to test and observe the effects of a series of actions that combine elements at the micro scale, experiment and anticipate future transformations.

The incorporation of "soft" data in dialogue with hard ones related to $\mathrm{CH}$, allows to ground the effective needs of local communities around heritage and to support and nurture the creation of a common sense of belonging in which citizens become agents and prosumers of the transformation, expressing needs, expectations, visions. This is only possible by providing a coherent picture of available resources (material and immaterial), trends and possible synergies, institutional and network initiatives. Therefore, the platform acquires relevance in the project, providing a shared digital environment where all stakeholders can contribute and at the same time be informed of progress. The use of a participatory process for the construction of local $\mathrm{CH}$ ontologies, conceived as site and community specific subsets of standardized $\mathrm{CH}$ makes it a replicable framework that could be employed elsewhere.

The system proposed by ROCK integrates a DW portion of the historic centre, with the proper dimension of interaction with the physical-relational dimension of urban space. In doing so, it seems more able to intercept the dynamics and contingencies of the urban spacetypically understood as elements that turn the digital model ineffective-rather incorporated as positive challenging elements to the model and feedbacks providing its flexibility. This allows to constantly actualize the data collected from the digital environment, intervening also on the dimension of response to contingency and, ultimately, to risk, hence useful to be prepared for future crisis.

The data collected and intercepted by the digital dimension of the urban model, requires clarity and interpretive-critical capacity, necessary for their transformation into design actions. This calls for the strengthening of an investigative and interactive action of administration and management of the city rather than a consultative and negotiation one. A position that sees in the role of the University another potential for active collaboration.

The ROCK platform's approach could be upscaled as a methodology to democratise the DW city paradigm not only by providing open data and user-friendly interfaces based on virtual reality and augmented reality, but through a collaborative design process. This collaboration can occur not only with the help of tech-savvy communities such as local coders and makers, but also through formal and informal association of citizens and stakeholders who do not possess specific IT skills. This approach to science, society and technologies, puts forward the collaboration around $\mathrm{CH}$ as a useful observation point for the verification and the strengthening of the policy assumptions of the New Green Deal and the New European Bauhaus.

\section{ACKNOWLEDGEMENTS}

The ROCK research project (https://rockproject.eu/) is co-financed by the European Union within the H2020 framework programme with Contract No. 730280. 


\section{REFERENCES}

[1] Townshend, T., and Pendlebury, J., Public participation in conservation. Journal of Urban Design, 4, pp. 313-331, 1999. https://doi.org/10.1080/13574809908724453.

[2] UNESCO, The UNESCO Recommendation on the Historic Urban Landscape, Online, available at http://unesdoc.unesco.org/images/0021/002150/215084e.pdf\#page $=52$ (accessed January 2021.)

[3] DC-NET, Service Priorities and Best Practices for Digital Cultural Heritage, Report, European Research Area Network, European Commission, Brussels, Online, available at https://www.digitalmeetsculture.net/article/dc-net-digital-cultural-heritage-network/, 2012 (accessed January 2021)

[4] European Commission, Commission Recommendation of 27 October 2011 on the digitisation and online accessibility of cultural material and digital preservation, Online, available at http://data.europa.eu/eli/reco/2011/711/oj (accessed January 2021).

[5] Turillazzi, B., Leoni, G., Gaspari, J., Iadanza, E., My, M., Massari, M., Boulanger, S.O.M. and Djalali, A., Heritage-led ontologies: digital platform for supporting the regeneration of cultural and historical sites. WIT Transactions on Ecology and the Environment, 249, pp. 307-318, 2020.

[6] European Expert Network on Culture and Audiovisual (EENCA), Platforms on the Future of Cultural Heritage: A problem solving approach Report on the Prague Platform on 'Cultural Heritage in the Digital Age', available at: https://eenca.com/eenca/assets/ File/EENCA\%20publications/Prague\%20Platform\%20Report\%20FINAL.pdf

[7] European Commission, Science and Society, available at https://ec.europa.eu/programmes/horizon2020/en/h2020-section/science-and-society (accessed April 2021).

[8] Sonkoly, Gábor and Vahtikari, Tanja (2018) Innovation in Cultural Heritage: For an Integrated European research policy. Working Paper. European Commission, Publications Office, Luxembourg, 53p. ISBN 978-92-79-78019-6.

[9] Reeve, Alan, and Shipley, R., Heritage-based regeneration in an age of austerity: Lessons from the Townscape Heritage Initiative. Journal of Urban Regeneration \& Renewal, 7.2, pp. 122-135, 2014. https://doi.org/10.1080/21650020.2014.893199

[10] Boeri, A., Borsari, A., Borghi, V., Gaspari, J., Gianfrate, V., Leoni, G., and Longo, D., Culture as Primary Political Action in City Governance: Three Key Concepts and Ten Policies to Start With. European Journal of Creative Practices in Cities and Landscapes 1, pp. 1-4, 2018.

[11] Getting cultural heritage to work for Europe, Report of the Horizon 2020 expert group on cultural heritage; Directorate-General for Research and Innovation, European Commission. Online, available at https://ec.europa.eu/programmes/horizon2020/en/ news/getting-cultural-heritage-work-europe (accessed January 2021).

[12] Owens, T., Digital cultural heritage and the crowd. Curator: The Museum Journal, 56(1), pp. 121-130. 2013.

[13] AA.VV, Declaration on joining forces to boost sustainable digital transformation in cities and communities in the EU, Online, available at https://www.living-in.eu/declaration (accessed January 2021).

[14] European Commission, The European Green Deal COM/2019/640 final, Online, available at https://eur-lex.europa.eu/legal-content/EN/TXT/?uri=CELEX:52019DC0640 (accessed 06 January 2021).

[15] ICOMOS Climate Change and Cultural Heritage Working Group. 2019. The Future of Our Pasts: Engaging Cultural Heritage in Climate Action, July 1, 2019. Paris: ICOMOS. Accessed 06 January 2021. 
[16] Adger, W. N., Barnett, J., Brown, K., Marshall, N., \& O'brien, K., Cultural dimensions of climate change impacts and adaptation. Nature climate change, 3(2), pp. 112-117, 2013.

[17] New European Bauhaus, available at https://europa.eu/new-european-bauhaus/system/files_en?file=2021-01/New-European-Bauhaus-Explained.pdf (accessed January 2021)

[18] Noh, Z., Sunar, M. S., and Pan, Z., A review on augmented reality for virtual heritage system. International conference on technologies for E-learning and digital entertainment, Springer, Berlin, Heidelberg, pp. 50-61, 2009. https://doi.org/10.1007/978-3642-03364-3_7.

[19] Borowiecki, K. J. Forbes, N., Fresa, A., Cultural heritage in a changing world. Springer Science+Business Media, 2016. https://doi.org/10.1007/978-3-319-29544-2

[20] Bonsma, Peter, et al. "Inception standard for heritage BIM models." Euro-Mediterranean Conference. Springer, Cham, 2016.

[21] Iadanza, E., et al. "Semantic web technologies meet BIM for accessing and understanding cultural heritage." 8th International Workshop 3D-ARCH 3D Virtual Reconstruction and Visualization of Complex Architectures". Vol. 42. Copernicus, 2019.

[22] Cooperation on advancing digitisation of cultural heritage, Digital Day 2019, Brussels, Belgium, 9 April 2019. Online, available at https://ec.europa.eu/digital-single-market/ en/news/eumember-states-sign-cooperate-digitising-cultural-heritage. (accessed January 2021).

[23] De Filippi, F., Coscia, C., \& Guido, R. (2020). Digital Platforms for Enhancing Participatory Design and Urban Regeneration: A Case Study in Turin (Italy). In CitizenResponsive Urban E-Planning: Recent Developments and Critical Perspectives (pp. 54-82). IGI Global.

[24] Longo, D., Boeri, A., Turillazzi, B., and Orlandi, S., Cultural heritage and interoperable open platforms: strategies for knowledge, accessibility, enhancement and networking. WIT Transactions on Ecology and the Environment, 241, pp. 371-382, 2020.

[25] Dembski, F., Wössner, U., Letzgus, M., Ruddat, M. and Yamu, C., Urban Digital Twins for Smart Cities and Citizens: The Case Study of Herrenberg, Germany. Sustainability, 12, pp. 2307, 2020. https://doi.org/10.3390/su12062307

[26] Grieves, M. and J. Vickers, Digital Twin: Mitigating Unpredictable, Undesirable Emergent Behavior in Complex Systems, in Trans-Disciplinary Perspectives on System Complexity, F.-J. Kahlen, S. Flumerfelt, and A. Alves, Editors. Springer: Switzerland, pp. 85-114, 2016.

[27] Dembski, Fabian, Wössner, Uwe and Letzgus, Mike. The Digital Twin Tackling Urban Challenges with Models, Spatial Analysis and Numerical Simulations in Immersive Virtual Environments, 2019. 10.5151/proceedings-ecaadesigradi2019_334.

[28] Dembski, F, Wössner, U and Yamu, C'Digital Twin, Virtual Reality and Space Syntax: Civic Engagement and Decision Support for Smart, Sustainable Cities', Proceedings of the 12th Space Syntax Symposium, Beijing, 2019.

[29] Farsi, M., Daneshkhah, A., Hosseinian-Far, A., Jahankhani, H. (Eds.). Digital Twin Technologies and Smart Cities. Springer, 2020

[30] Boeri, A., Gaspari, J., Gianfrate, V., Longo, D., and Boulanger, S.O.M., Circular City: a methodological approach for sustainable districts and communities. Eco-Architecture VII: Harmonisation between Architecture and Nature, 183, pp. 73-82, 2019. https://doi. org/10.2495/ARC180071. 
[31] Gianfrate, V., Djalali, A., Turillazzi, B., Boulanger, S.O.M., Massari, M., Research-actionresearch towards a circular urban system for multi-level regeneration in historical cities: The case of Bologna. International Journal of Design \& Nature and Ecodynamics, Vol. 15, No. 1, pp. 5-11, 2020. https://doi.org/10.18280/ijdne.150102.

[32] Boeri, A., Gaspari, J., Gianfrate, V., Longo, D., and Pussetti, C., The adaptive reuse of historic city centres. Bologna and Lisbon: solutions for urban regeneration. TECHNEJournal of Technology for Architecture and Environment, 12, pp. 230-237, 2016.

[33] Boeri, A., Gaspari, J., Gianfrate, V., Longo, D., and Boulanger, S., Circular city: A methodological approach for sustainable districts and communities. Eco-Architecture VII: Harmonisation between Architecture and Nature, 183, pp. 73, 2019.

[34] Boeri A., Longo D., Gianfrate V., Roversi R. and Boulanger S. Co-designing the Urban Accessibility. An Inclusive Fruition Service in the Bologna University Area, in: Abitare inclusivo: il progetto per una vita autonoma e indipendente. Inclusive living: design for an autonomous and independent living, Conegliano - TV, Anteferma Edizioni S.r.1., pp. 92-101, 2019.

[35] Longo D., Gianfrate V., and Massari M., Il Progetto europeo ROCK. La città come laboratorio di conoscenza e innovazione, in: Spazi ed educazione, Canterano (RM), Aracne editrice, pp. 197-215, 2019.

[36] Leminen, S., Rajahonka, M., and Westerlund, M., Towards Third-Generation Living Lab Networks in Cities. Technology Innovation Management Review, 7(11), pp. 21-35, 2017. https://doi.org/10.22215/timreview/1118

[37] Dane, G. Z., Derakhshan, S., Ettefagh, T., Massari, M., Gianfrate, V., and Bigi, M. Participatory mapping of citizens' experiences at public open spaces: a case study at Bologna living lab. In Proceedings of 25th International Conference on Urban Planning and Regional Development in the Information Society GeoMultimedia 2020 (pp. 645654), 2020, https://archive.corp.at/cdrom2020/papers2020/CORP2020_91.pdf 